\title{
Electroacupuncture alleviates polycystic ovary syndrome-like symptoms through improving insulin resistance, mitochondrial dysfunction, and endoplasmic reticulum stress via enhancing autophagy in rats
}

\author{
Yan Peng ${ }^{1 \dagger}$, Liyuan Guo ${ }^{2 \dagger}$, Anxin $\mathrm{Gu}^{3}$, Beibei Shi ${ }^{4}$, Yukun Ren ${ }^{4}$, Jing Cong $^{5}$ and Xinming Yang ${ }^{5^{*}}$ (D)
}

\begin{abstract}
Background: Electroacupuncture (EA), a treatment derived from traditional Chinese medicine, can effectively improve hyperandrogenism and insulin resistance in patients with polycystic ovary syndrome (PCOS), however, its underlying mechanisms remain obscure. This study aimed to investigate whether EA could mitigate PCOS-like symptoms in rats by regulating autophagy.

Methods: A rat model of PCOS-like symptoms was established by subcutaneous injection with dehydroepiandrosterone (DHEA), and then EA treatment at acupoints (ST29 and SP6) was carried out for 5 weeks. To inhibit autophagy in rats, intraperitoneal injection with $0.5 \mathrm{mg} / \mathrm{kg}$ 3-MA (an autophagy inhibitor) was performed at 30 min before each EA treatment.

Results: EA intervention alleviated PCOS-like symptoms in rats, which was partly counteracted by the combination with 3-MA. Moreover, DHEA-exposure-induced deficient autophagy in skeletal muscle was improved by EA treatment. EA-mediated improvements in insulin resistance, mitochondrial dysfunction, and endoplasmic reticulum (ER) stress in PCOS-like rats were counteracted by 3-MA pretreatment. Mechanically, EA attenuated autophagy deficiency-mediated insulin resistance in PCOS-like rats via inactivating mTOR/4E-BP1 signaling pathway.

Conclusions: Taken together, our findings indicate that EA treatment ameliorates insulin resistance, mitochondrial dysfunction, and ER stress through enhancing autophagy in a PCOS-like rat model. Our study provides novel insight into the mechanisms underlying the treatment of EA in PCOS, which offers more theoretic foundation for its clinical application.
\end{abstract}

Keywords: Electroacupuncture, Polycystic ovary syndrome, Insulin resistance, Autophagy, ER stress, Mitochondrial dysfunction

\footnotetext{
*Correspondence: yxm1980123@163.com

${ }^{\dagger}$ Yan Peng and Liyuan Guo contributed equally to this work.

${ }^{5}$ Department of Obstetrics and Gynecology, The First Affiliated Hospital

Heilongjiang University of Chinese Medicine, Harbin 150040, People's

Republic of China

Full list of author information is available at the end of the article
}

(c) The Author(s). 2020 Open Access This article is licensed under a Creative Commons Attribution 4.0 International License, which permits use, sharing, adaptation, distribution and reproduction in any medium or format, as long as you give appropriate credit to the original author(s) and the source, provide a link to the Creative Commons licence, and indicate if changes were made. The images or other third party material in this article are included in the article's Creative Commons licence, unless indicated otherwise in a credit line to the material. If material is not included in the article's Creative Commons licence and your intended use is not permitted by statutory regulation or exceeds the permitted use, you will need to obtain permission directly from the copyright holder. To view a copy of this licence, visit http://creativecommons.org/licenses/by/4.0/ 


\section{Introduction}

Polycystic ovary syndrome (PCOS) also known as SteinLeventhal syndrome, is a complex endocrine and metabolic disorder in gynecology and mainly characterized by hyperandrogenism and insulin resistance (Stener-Victorin et al. 2016). Its incidence is $5-10 \%$ among women of reproductive age, which is higher than other infertilities (Batista et al. 2012; Goodarzi et al. 2011). It has been shown that insulin resistance, an important pathological characteristic of PCOS, has been observed in nearly 85\% PCOS patients (Stepto et al. 2013). At present, antiestrogen agents are the first-line drugs for treating infertility in women with PCOS. However, these drugs may frequently lose effectiveness and even cause adverse effects (Legro et al. 2007; Zain et al. 2009).

A great deal of animal and human studies proved that acupuncture treatment could effectively relieve the symptoms of PCOS. For example, electroacupuncture (EA) has been shown to modulate the circulating gonadotropin levels and ovarian adiponectin system in PCOS-like rats (Maliqueo et al. 2015). Johansson et al. found that EA intervention could increase ovulation frequency in patients with PCOS (Johansson et al. 2013). Besides, EA has been verified to improve hyperandrogenism and follicular arrest in PCOS-like animals (Shi et al. 2019). In addition, mounting evidence has proved that insulin resistance can be attenuated by EA treatment in PCOS (Johansson et al. 2010; Stener-Victorin et al. 2012; Stener-Victorin et al. 2016). Notwithstanding all this, the detailed mechanisms of EA underlying the protection against PCOS remain ambiguous.

Autophagy dysfunction has been confirmed to contribute to the pathogenesis of PCOS. A previous study showed that the levels of autophagy-related protein DNA damage regulated autophagy modulator 2 was declined in the granulosa cells of PCOS patients (Dai and Lu 2012). The levels of autophagy-related genes ATG14, Beclin-1, and ATG-3 were reduced in the endometrium of PCOS patients as compared with the normal controls, which could be remarkably enhanced by metformin treatment (Sumarac-Dumanovic et al. 2017). Moreover, it has been shown that the promotion of autophagy could improve insulin resistance in nonalcoholic steatohepatitis (Amir and Czaja 2011), suggesting that modulation of autophagy tightly connected to insulin resistance. Additionally, overexpression of key autophagy regulatory protein mechanistic target of rapamycin kinase (mTOR) can lead to insulin resistance during the pathological development of PCOS (Liu et al. 2018; Song et al. 2018). Besides, mitochondrial dysfunction and ER stress have been shown to participate in the pathogenesis of PCOS (Azhary et al. 2020; Zeng et al. 2020). Previous studies suggested that autophagy was closely related to mitochondrial dysfunction (Go et al. 2015) and ER stress (Lee et al. 2015). Currently, it is not clear whether EA can mitigate insulin resistance, mitochondrial dysfunction and ER stress through regulating autophagy in PCOS.

In this study, a PCOS-like rat model was established by dehydroepiandrosterone (DHEA)injection. EAmediated modulation of autophagy and its involvements in insulin resistance, mitochondrial dysfunction, and ER stress in PCOS-like rats were further investigated. Our findings shed light on the novel protective mechanisms of EA in treating PCOS.

\section{Methods}

\section{Animal model}

Four-week-old female Sprague-Dawley (SD) rats were purchased from Chang Sheng biotechnology co., Ltd. (Liaoning, China) and randomly divided into four experimental groups ( $n=12$ per group): control group, PCOS group, PCOS+EA group, and PCOS+EA + 3-MA group. PCOS-like symptoms in rats were induced by daily subcutaneous injection with DHEA (Meilunbio, Dalian, China) at a dose of $6 \mathrm{mg} / 100 \mathrm{~g}$ body weight for 20 consecutive days. The control rats were subcutaneously injected with equal volume sesame oil daily. Immediately after the first injection with DHEA, EA treatment was performed every other day for 5 weeks. According to a previous study (Manneras et al. 2008), the acupuncture points ST29 and SP6 were electrically stimulated at a frequency of $2 \mathrm{~Hz}$ and intensity of $0.8-1.3 \mathrm{~mA}$. The EA duration was $15 \mathrm{~min}$ in week $1,20 \mathrm{~min}$ in week $2-3$, and $25 \mathrm{~min}$ in week 4-5. The rats in PCOS+EA + 3-MA group were received intraperitoneal injection with 0.5 $\mathrm{mg} / \mathrm{kg}$ 3-MA (Aladdin, Shanghai, China) at $30 \mathrm{~min}$ before each EA treatment. All rats were subjected to a 12$h$ fast at the night before the end of the experiment. For each group, six rats were received intraperitoneal injection of $1 \mathrm{U} / \mathrm{kg}$ insulin (Aladdin), while the other six rats were injected with equal volume normal saline. $15 \mathrm{~min}$ after the injection, all rats were euthanized and then the blood sample, ovary and skeletal muscle tissues were collected for further experiments.

\section{HE staining}

The ovary tissues were fixed in $4 \%$ paraformaldehyde, immersed in alcohol gradient, and then embedded in paraffin, followed by cutting into $5-\mu \mathrm{m}$ sections. After dewaxing and hydration, the sections were successively stained with hematoxylin for $5 \mathrm{~min}$ and eosin for $3 \mathrm{~min}$. Finally, the sections were observed under a microscope (Olympus, Japan) at a magnification of $100 \times$.

\section{Elisa}

The serum levels of testosterone, follicle stimulating hormone (FSH), luteal hormone ( $\mathrm{LH})$, and insulin were 
determined using the commercial ELISA kits (USCN Life Science, Wuhan, China) according to the manufacturer's protocol.

\section{Real-time PCR}

Total RNA was extracted from ovary or skeletal muscle tissues using a RNApure total RNA isolation kit (Bio Teke, Beijing, China). The concentration of RNA was detected on an ultraviolet spectrophotometer (Nana Drop 2000, Thermo Scientific, USA). Subsequently, cDNA was synthesized using M-MLV Reverse Transcriptase (Takara, Japan). Real time PCR was carried out using Taq HS Perfect Mix (Takara) and SYBR Green (Bio Teke) on an Exicycler ${ }^{\text {Tu }} 96$ Real-Time Quantitative Thermal Block (BIONEER, Korea). The primer sequences are presented in Table 1 . The mRNA expression level was calculated by $2^{-\triangle \Delta C T}$ method.

\section{Western blotting}

Total protein was extracted from ovary and skeletal muscle tissues using RIPA buffer (Beyotime, china) supplemented with 1\% PMSF (Beyotime). Protein quantification was performed using an Enhanced BCA Protein Assay Kit (Beyotime). Sodium dodecyl sulfate polyacrylamide gel electrophoresis was carried out for protein separation. Then the protein samples were transferred onto PVDF membrane (Thermo Scientific) and blocked in 5\% BSA (Biosharp, China). Subsequently, the membranes were incubated with the primary antibodies against CYP17 (1:500, Proteintech, China), CYP19 (1: 500, Proteintech), LC3I/II (1:1000, Cell Signaling Technology, USA), Beclin-1 (1:1000, Proteintech), p62 (1: 2000, Proteintech), p- mTOR (1:1000, Cell Signaling Technology), mTOR (1:1000, Cell Signaling Technology), 4E-BP1 (1:1000, Cell Signaling Technology), p-4EBP1 (1:1000, Cell Signaling Technology), PGC-1 $\alpha$ (1: 1000, Affinity, China), PPARY (1:1000, Proteintech), GLUT4 (1:500, Proteintech), p-AKT (1:2000, Cell Signaling Technology), AKT $(1: 1000, \quad$ Cell Signaling

Table 1 Oligonucleotide primer sets for real-time PCR

\begin{tabular}{|c|c|c|}
\hline Name & Sequence $\left(5^{\prime} \neg 3^{\prime}\right)$ & Length \\
\hline CYP17 F & TGGAGGTGATAAAGGGTT & 18 \\
\hline CYP17 R & CGTCAGGCTGGAGATAGA & 18 \\
\hline CYP19 F & GCCTGTCGTGGACTTGGT & 18 \\
\hline CYP19R & TAAATTCATTGGGCTTGG & 18 \\
\hline PPARY F & TACCACGGTTGATTTCTC & 18 \\
\hline PPARY R & AATAATAAGGCGGGGACG & 18 \\
\hline PGC-1a F & GAACAAGACTATTGAGCGAACC & 22 \\
\hline PGC-1aR & GAGTGGCTGCCTTGGGTA & 18 \\
\hline$\beta$-actin $F$ & CCACTGCCGCATCCTCTT & 18 \\
\hline$\beta$-actin R & GGTCTTTACGGATGTCAACG & 20 \\
\hline
\end{tabular}

Technology), p-ERK (1:2000, Cell Signaling Technology), ERK (1:1000, Cell Signaling Technology), cytochrome C (1:1000, Abclonal, China), GRP78 (1:1000, Abclonal), ATF4 (1:1000, Abclonal), CHOP (1:1000, Abclonal, China) GRP78 (1:1000, Abclonal), and $\beta$-actin (1:2000, Proteintech) at $4{ }^{\circ} \mathrm{C}$ overnight. Then the membranes were incubated with Goat anti-rabbit or mouse IgG (1: 10000 , Proteintech) at $37^{\circ} \mathrm{C}$ for $40 \mathrm{~min}$. ECL reagent (7 sea biotech, China) was used to visualize the protein bands.

\section{Isolation of plasma membrane from skeletal muscle for testing GLUT4 translocation}

The plasma membrane of the skeletal muscle tissues was isolated as previously described (Wang et al. 2018). Briefly, the skeletal muscle tissues were rinsed in Solution A containing $250 \mathrm{mmol} / \mathrm{L}$ Sucrose, $50 \mathrm{mmol} / \mathrm{L}$ Tris, $0.2 \mathrm{mmol} / \mathrm{L}$ EDTA, $\mathrm{pH} 7.4$, followed by centrifugation at $120 \mathrm{~g}$ for $15 \mathrm{~min}$ at $4{ }^{\circ} \mathrm{C}$. Then the mixed supernatant was collected and centrifuged at $9000 \mathrm{~g}$ for $20 \mathrm{~min}$ at $4{ }^{\circ} \mathrm{C}$. Subsequently, the supernatant was collected and centrifuged at $190,000 \mathrm{~g}$ for $60 \mathrm{~min}$ at $4{ }^{\circ} \mathrm{C}$. The resulting pellets were re-suspended in $25 \%$ sucrose solution and centrifuged at $150,000 \mathrm{~g}$ for $16 \mathrm{~h}$ at $4{ }^{\circ} \mathrm{C}$. The $25 \%$ sucrose layer was collected and centrifuged at $190,000 \mathrm{~g}$ for $1 \mathrm{~h}$ at $4{ }^{\circ} \mathrm{C}$. The resulting pellets were re-suspended with $100 \mu \mathrm{L}$ of Solution A. Finally, the samples were subjected to Western blotting for testing GLUT4 translocation.

\section{Detection of mitochondrial complex enzymes}

The activities of mitochondrial complex I and III in the ovarian tissues of rats were assessed using the commercial mitochondrial respiratory chain complex I and III assay kits (Solarbio) following the manufacturer's instructions.

\section{Immunofluorescence staining}

To assess LC3 expression in skeletal muscle tissues, immunofluorescence staining was carried out. Briefly, the paraffin-embedded skeletal muscle tissues were successively heated in an oven at $60^{\circ} \mathrm{C}$ for $30 \mathrm{~min}$, immersed in xylene for $15 \mathrm{~min}$, and hydrated in gradient ethanol. After antigen retrieval and blocking in goat serum (Solarbio, China), the sections were incubated with primary antibody LC3 $\left(1: 100\right.$, Proteintech) at $4{ }^{\circ} \mathrm{C}$ overnight, followed by incubation with Cy3-labeled Goat Anti-Rabbit IgG (1:200, Beyotime) for $60 \mathrm{~min}$. After nuclear staining with DAPI, the sections were observed under an inverted fluorescence microscope (Olympus, Japan). 


\section{Statistical analysis}

All experimental data expressed as mean \pm standard deviation were statistically analyzed using one-way analysis of variance followed by Tukey post-tests. Significance was recognized as a $P$ value less than 0.05 .

\section{Results \\ Inhibition of autophagy reversed the beneficial effect of EA on PCOS-like rats}

As shown in Fig. 1a, the pathological manifestations of ovarian tissues after exposure to DHEA were determined by HE staining. The number of follicular cysts of multiple sizes was significantly increased in the ovarian tissues of DHEA-exposed rats, as compared with that of control rats. However, EA treatment could remarkably reduce the number of follicular cysts in PCOS-like rats, which was reversed by autophagy inhibitor 3-MA administration. In addition, the serum levels of testosterone, $\mathrm{LH}$ and $\mathrm{LH} / \mathrm{FSH}$ ratio in PCOS-like rats were remarkably elevated, while the serum FSH level was reduced by almost half (Fig. 1-B-E). Whereas, these changes were attenuated by EA intervention, which were partly restored by 3-MA treatment. Moreover, the increased mRNA levels of CYP17 and CYP19 in the ovarian tissues of PCOS-like rats were downregulated by EA treatment (Fig. 1f\&g). As expected, suppression of autophagy by 3-MA reversed EA-mediated changes in CYP17 and CYP19 mRNA levels. These data indicated that EA attenuated PCOS-like symptoms in rats via promoting autophagy.

\section{Effect of EA on autophagy in PCOS-like rats}

Since autophagy promotion participated in the protection of EA against PCOS, the regulation of EA in autophagy was further investigated in PCOS-like rats. As presented in Fig. 2a-c, DHEA exposure-induced upregulation of LC3II/I ratio and Beclin-1 level, but downregulation of p62 level in skeletal muscle tissues were restrained by EA treatment, which were suppressed by 3-MA administration. Moreover, the expression of LC3 in the skeletal muscle tissues was determined by immunofluorescent staining (Fig. 2d). DHEA-challenged rats exhibited lower expression of LC3 in the skeletal muscle tissues compared with control rats, indicating defective autophagy in PCOSlike rats. Treatment with EA significantly restored autophagy by increasing LC3 expression, which was effectively restrained by 3 -MA. Therefore, EA treatment promoted autophagy in PCOS-like rats.

\section{EA restrained insulin resistance in PCOS-like rats via enhancing autophagy}

Since insulin resistance is one of important pathological features of PCOS, we further investigated the involvement of autophagy in EA-mediated insulin resistance. As shown in Fig. 3a, the fasting serum insulin level of PCOSlike rats was increased than that in control group, which was effectively downregulated by EA treatment. 3-MA administration repressed EA-mediated downregulation of insulin level. Furthermore, EA intervention led to decreased HOMA-IR index of PCOS-like rats, which was restrained by 3-MA (Fig. 3b). Additionally, insulin sensitivity was evaluated by detecting insulin signaling protein levels in rats treated with or without insulin. As shown in Fig. 3c-f, the protein levels of GLUT4, p-AKT, and p-ERK were obviously decreased in the skeletal muscle tissues of PCOSlike rats. As might be expected, EA treatment could upregulate GLUT4 and p-ERK levels, and 3-MA suppressed these changes. There was no statistical difference in $\mathrm{p}$ AKT level, although p-AKT level appeared to be raised after EA treatment. Insulin-stimulated the translocation of GLUT4 was inhibited by DHEA exposure. However, EA intervention restored GLUT4 translocation in response to insulin, which was reversed by 3-MA administration (Fig. $3 g$ ). These results suggested that EA treatment restrained DHEA-induced insulin resistance in skeletal muscle tissues of PCOS-like rats through enhancing autophagy.

\section{EA attenuated insulin resistance via regulating $\mathrm{mTOR} / 4 \mathrm{E}-$ BP1 signaling pathway}

As presented in Fig. 4a\&b, EA treatment remarkably enhanced the mRNA expression of insulin resistancerelated factors PGC- $1 \alpha$ and PPAR $\gamma$ in PCOS-like rats. Similarly, EA treatment increased the protein levels of PGC-1 $\alpha$ and PPAR $\gamma$ in the skeletal muscle tissues of rats exposed to DHEA (Fig. 4C\&D). Furthermore, DHEA exposure-induced upregulation of $\mathrm{p}-\mathrm{mTOR}$ and $\mathrm{p}-4 \mathrm{E}-$ BP1 levels was inhibited by EA treatment (Fig. 4E\&F).

\section{EA improved mitochondrial dysfunction in PCOS-like rats via enhancing autophagy}

Next, we investigated the improvement in mitochondrial dysfunction underlying the beneficial effects of EA on PCOS. As presented in Fig. 5A\&B, the reduced activities of Complex I and Complex III in the ovarian tissues of PCOS-like rats were evidently improved by EA treatment. Whereas, 3-MA-mediated suppression of autophagy partly reversed the improvement in Complex I and Complex III activities in EA-treated rats. Moreover, Western blotting results showed that EA intervention restrained cytochrome $\mathrm{C}$ release from mitochondria into cytoplasm in PCOS-like rats, which was counteracted by 3-MA administration (Fig. 5C\&D). Thus, EA improved mitochondrial dysfunction in PCOS-like rats via activating autophagy.

\section{EA attenuated ER stress in PCOS-like rats via promoting autophagy}

To further explore whether EA alleviated PCOS-like symptoms in rats via relieving ER stress, the protein 


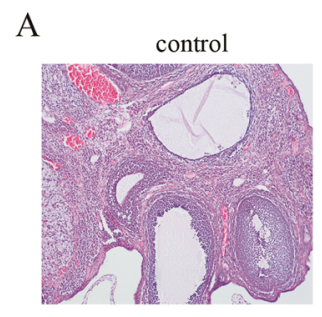

B
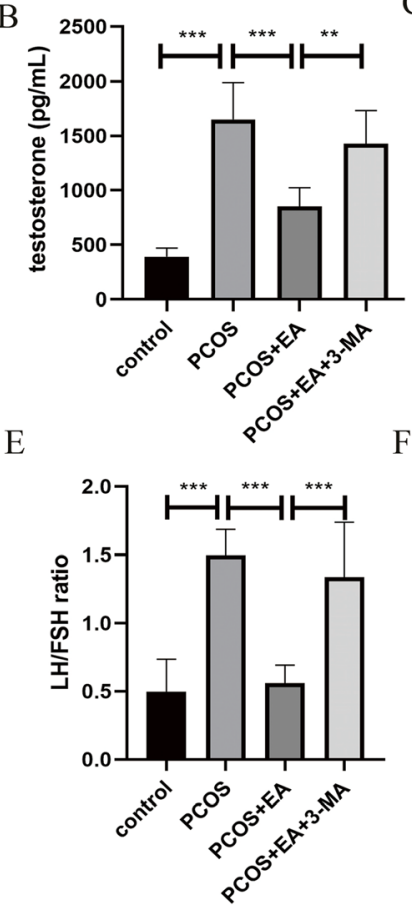

$\mathrm{H}$

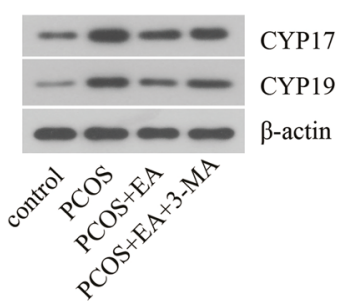

PCOS

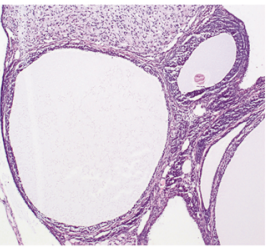

$\mathrm{C}$

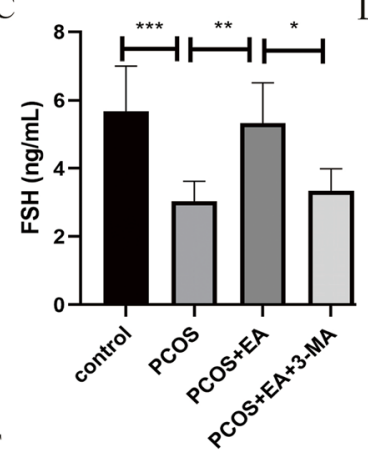

D
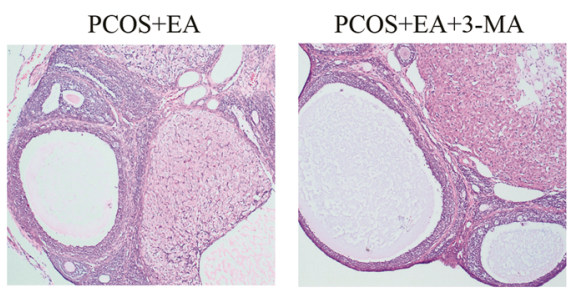

6000

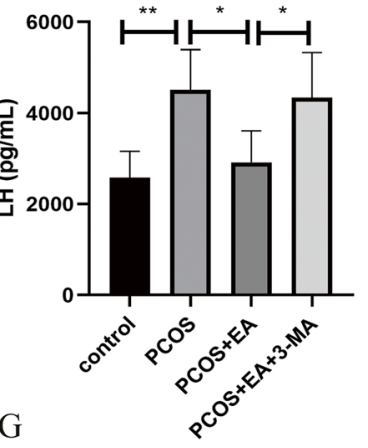

G
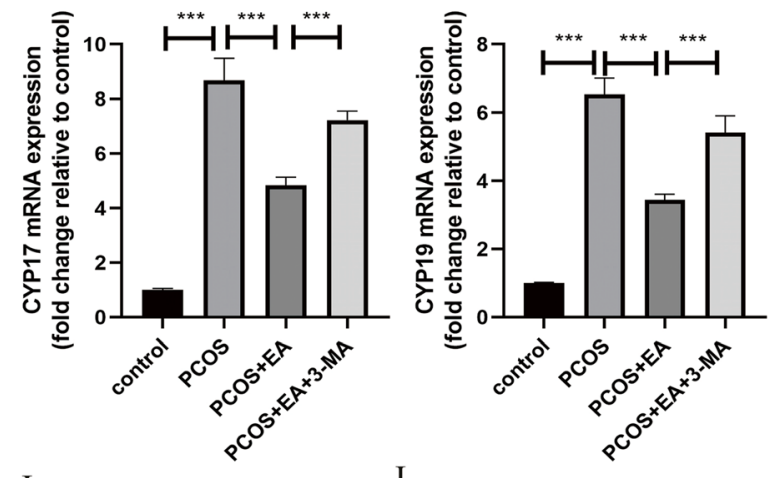

I
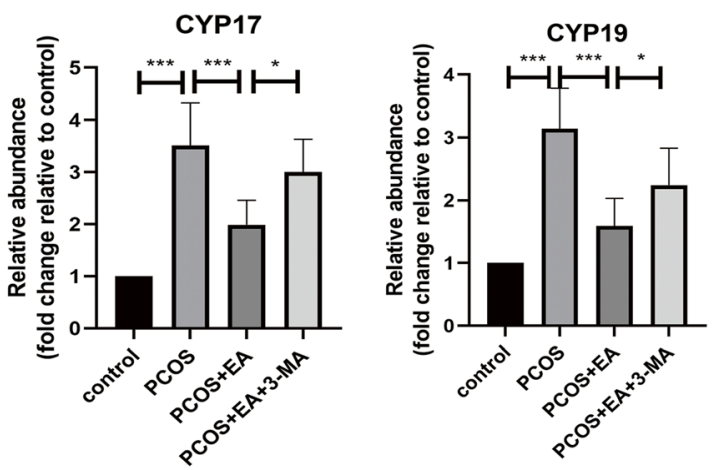

Fig. 1 Autophagy inhibition repressed the beneficial effect of EA on PCOS-like rats. a The pathological changes of ovarian tissues were determined by HE staining (100x). The serum levels of testosterone (b), FSH (c), and LH (d) were assessed by commercial ELISA kits. (e) The ratio of LH/FSH was calculated and shown. The mRNA expression of CYP17 (f) and CYP19 (g) was detected by real-time PCR. (h-j) The protein levels of CYP17 and CYP19 were evaluated by Western blotting. The experimental data are presented as mean \pm standard deviation $(n=6) .{ }^{*} P<0.05$, ${ }^{* *} P<0.01,{ }^{* * *} P<0.001$, vs the indicated group. EA, electroacupuncture; PCOS, polycystic ovary syndrome; FSH, follicle stimulating hormone; LH, luteal hormone

levels of ER stress-related proteins GRP78, ATF4, and $\mathrm{CHOP}$ were detected by Western blotting. As shown in Fig. 6A-C, a significant increase in the protein levels of GRP78, ATF4, and CHOP in the ovarian tissues of PCOS-like rats was observed, which was declined after receiving EA treatment. Whereas, EA-mediated decrease in GRP78, ATF4, and CHOP levels was significantly repressed by combination with 3-MA. These findings indicated that EA relieved ER stress via promoting autophagy in PCOS-like rats. 


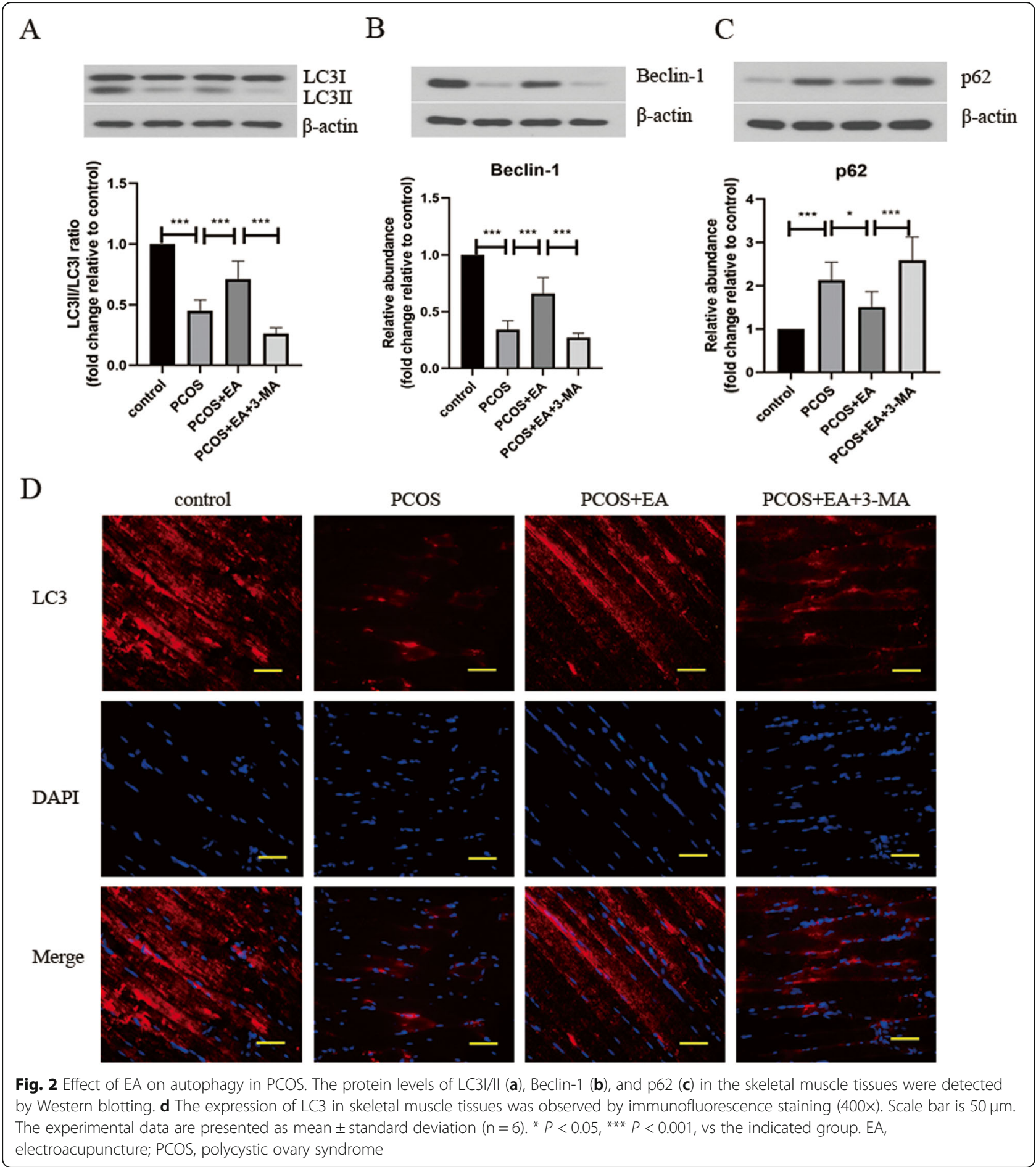

\section{Discussion}

PCOS is a complicated disorder of reproductive and endocrine system, which causes serious harm to women all over the world (Moran and Teede 2009). Hyperandrogenism and insulin resistance contribute to the reproductive abnormity in PCOS patients (Rosenfield and Ehrmann 2016). Plenty of clinical trials have suggested that acupuncture may be an effective treatment for PCOS (Cao et al. 2019; Stener-Victorin et al. 2019; Wang et al. 2019). However, the potential mechanisms of acupuncture underlying the treatment of PCOS have not been fully illuminated. In this study, 3-MA-mediated autophagy inhibition counteracted the beneficial effects of EA on PCOS-like symptoms in rats. In addition, our 


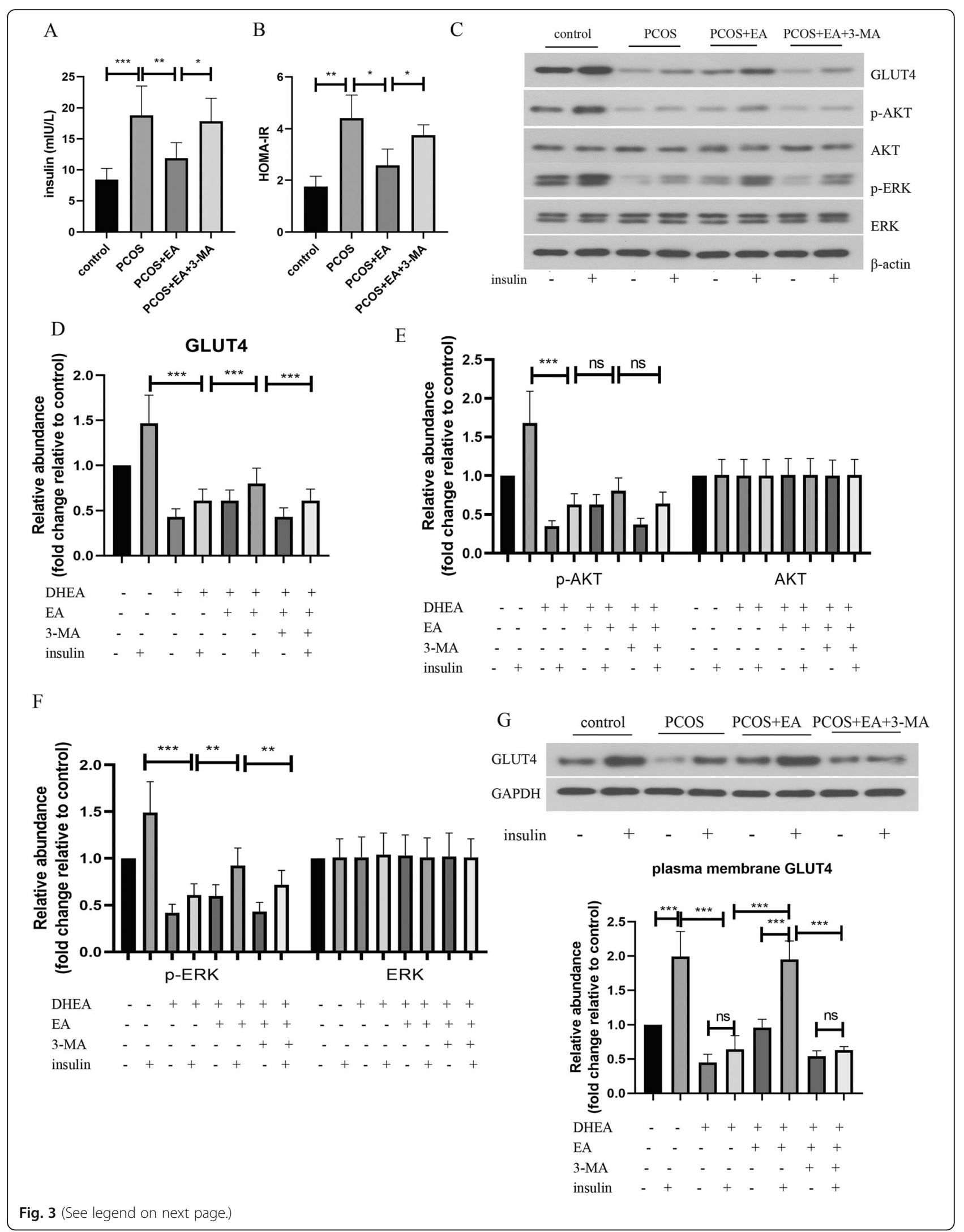


(See figure on previous page.)

Fig. 3 EA restrained insulin resistance in PCOS-like rats via enhancing autophagy. a The serum insulin level was detected by a commercial ELISA kit. $\mathbf{b}$ The HOMA-IR index was detected and shown. c-f The protein levels of GLUT4, p-AKT, AKT, p-ERK, and ERK in skeletal muscle tissues were determined by Western blotting. $\mathbf{g}$ Expression of GLUT4 in plasma membrane of skeletal muscle tissues was detected by Western blotting. The experimental data are presented as mean \pm standard deviation $(n=6)$. ${ }^{*} P<0.05,{ }^{*} P<0.01,{ }^{* * *} P<0.001$, vs the indicated group. PCOS,

polycystic ovary syndrome; HOMA-IR, homeostasis model assessment of insulin resistance

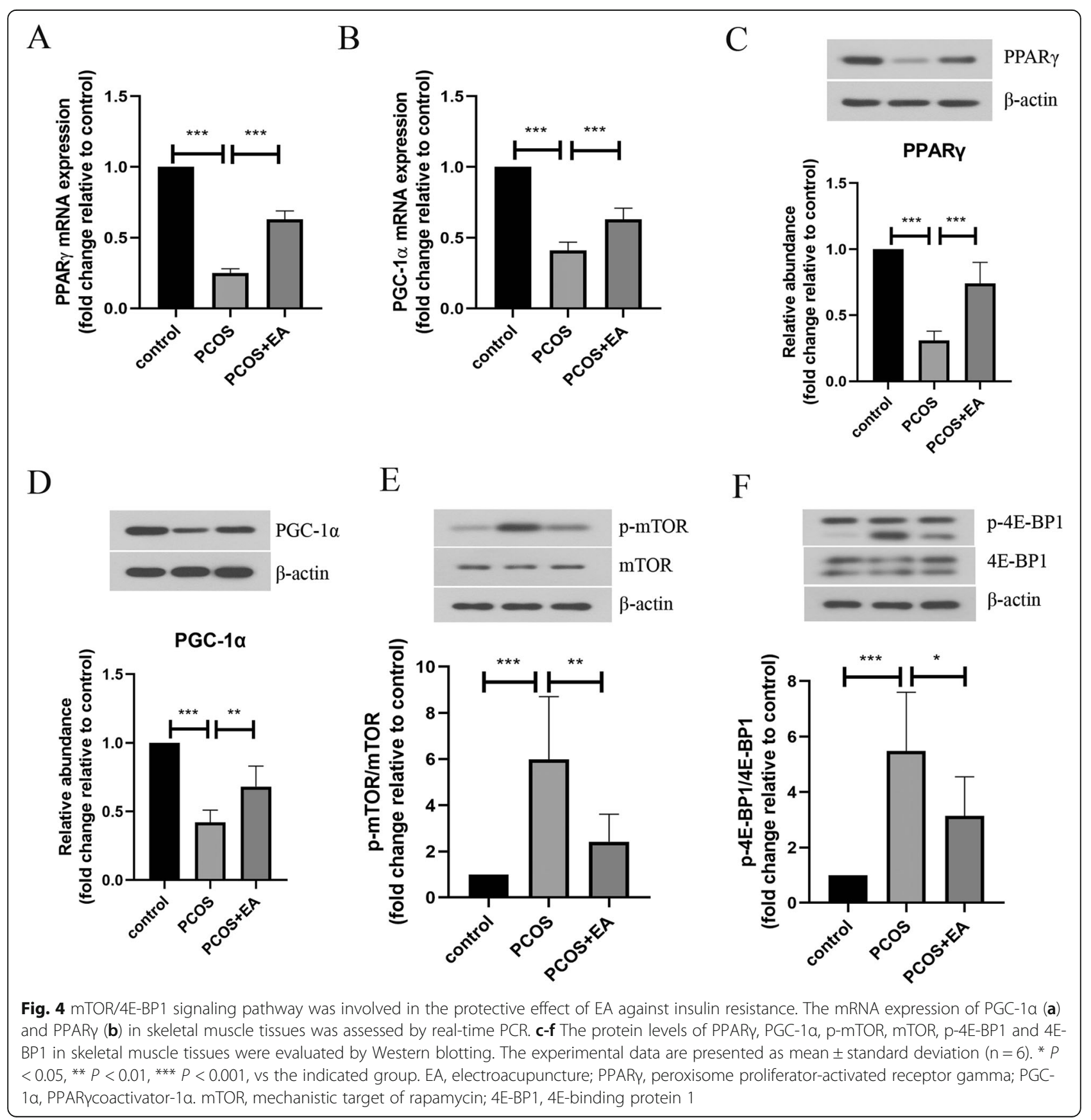




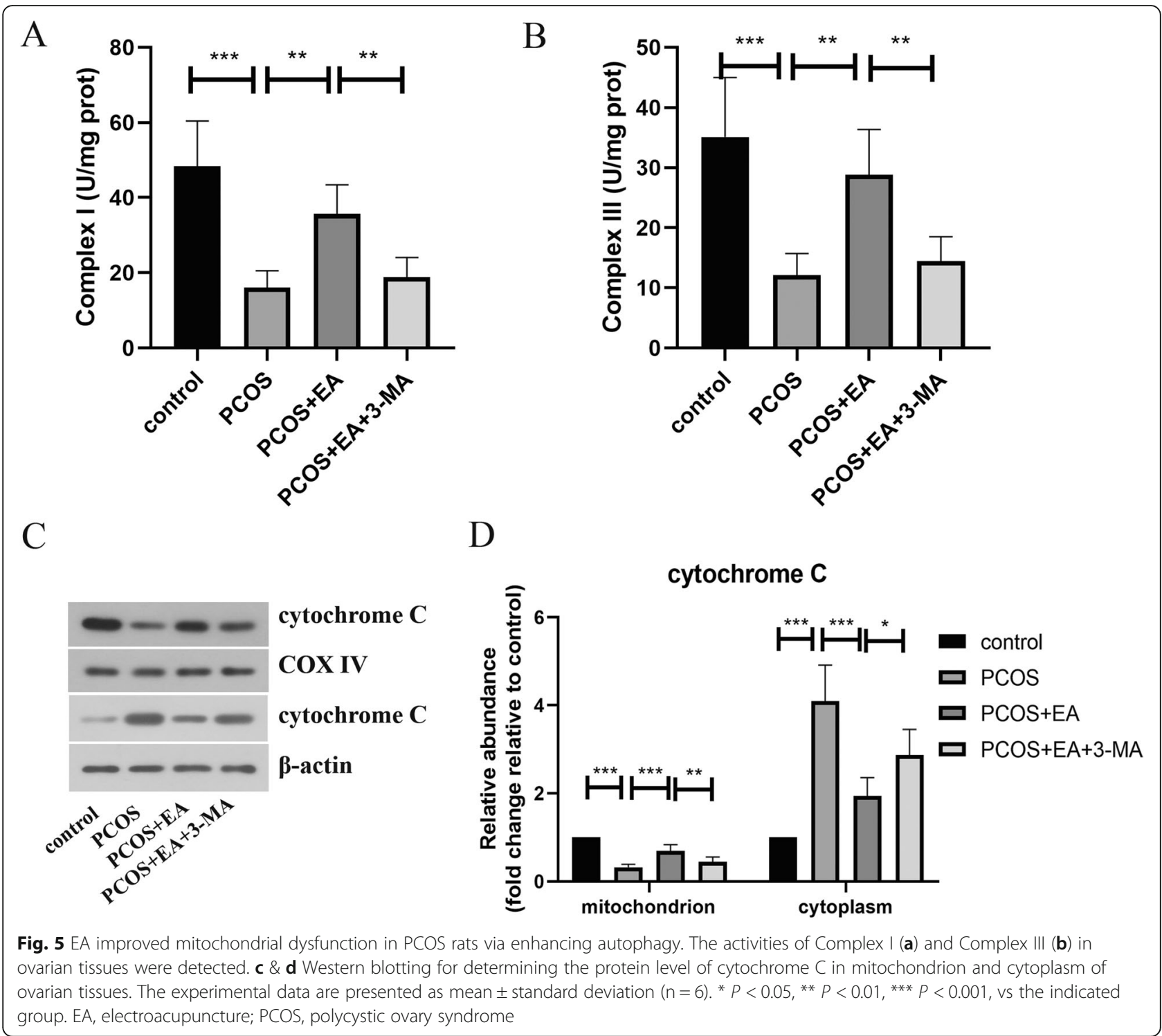

results for the first time demonstrated that the defective autophagy in PCOS-like rats was activated by EA intervention. Moreover, EA ameliorated insulin resistance, mitochondrial dysfunction, and ER stress induced by DHEA via promoting autophagy. Mechanically, EA treatment activated autophagy via regulating $\mathrm{mTOR} / 4 \mathrm{E}-$ BP1 signaling pathway.

Previous researches have demonstrated that DHEAinduced PCOS-like animal model displays the clinical characteristics of human PCOS, including hyperandrogenism, aberrant maturation of ovarian follicles and anovulation (Anderson et al. 1992; Lee et al. 1998; Luchetti et al. 2004). A study by Xi et al. showed that DHEAexposed mice presented whole-body and skeletal muscle insulin resistance, along with autophagy inhibition and mitochondrial injury (Song et al. 2018). Therefore, we selected DHEA to establish a rat model of PCOS-like symptoms, and investigated the role of autophagy in EAmediated protection against PCOS.

A number of rodent and human studies have proved the effectiveness of EA in treating PCOS. For example, low-frequency EA reversed the epigenetic and transcriptional changes in the adipose tissues of PCOS patients (Kokosar et al. 2018). Electrical or manual acupuncture improved insulin sensitivity by regulating the expression of multiple metabolic Genes and signaling pathways in PCOS model (Benrick et al. 2014). EA enhanced wholebody glucose uptake by activating nervous systems of PCOS women (Benrick et al. 2017). Even so, the detailed mechanisms of EA underlying the protection against PCOS need to be further investigated. Autophagy, as is a physiological process, participates in the pathological processes of multiple diseases, including PCOS. It is reported that the expression of autophagy-related genes 


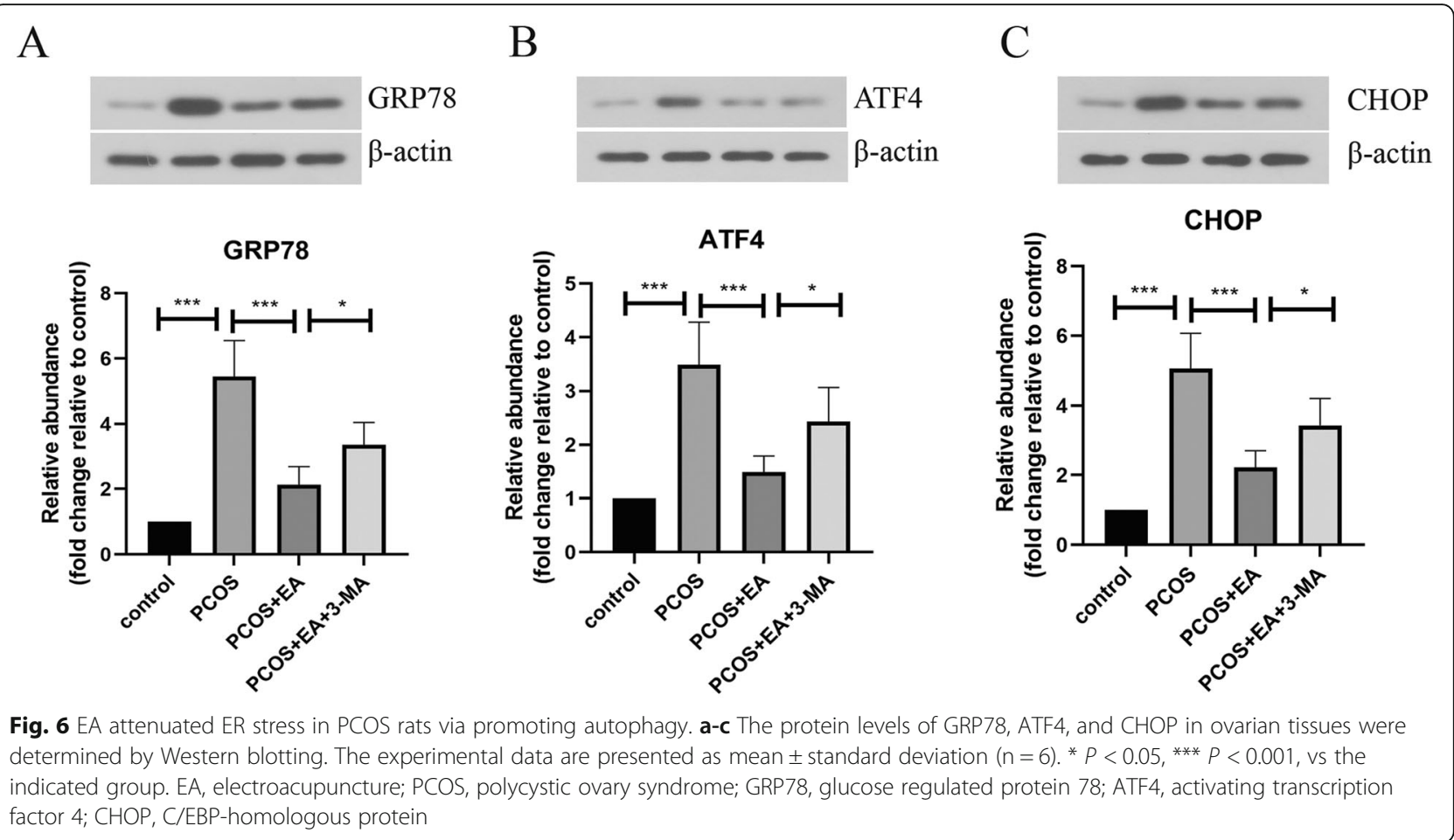

was significantly affected in the endometrium of PCOS patients (Sumarac-Dumanovic et al. 2017). Li et al. suggested that autophagy was abnormally activated in ovarian granulosa cells from PCOS patients ( $\mathrm{Li}$ et al. 2019). Consistently, another research demonstrated the activation of autophagy in PCOS patients and rats ( $\mathrm{Li}$ et al. 2018). At present, the regulation of autophagy in PCOS is still controversial. In the present study, a decrease in LC3II and Beclin-1 expression, while an increase in p62 expression were found in the skeletal muscle tissues of PCOS-like rats, indicating a suppression of autophagy in PCOS-like rats. Previous studies have reported the regulation of autophagy by acupuncture. For example, EA preconditioning-mediated autophagy promotion could relieve myocardial infarction (Zeng et al. 2018). In addition, in a rat model of Parkinson's disease, EA treatment could enhance autophagy ( $\mathrm{Li}$ et al. 2017). In this study, we provided first evidence that EA treatment remarkably enhanced autophagy in the skeletal muscle of PCOS-like rats. Further experiments demonstrated that EA-mediated relief of the hyperandrogenism was restrained by autophagy inhibition. Thus, our data demonstrated that EA alleviated PCOS-like symptoms in rats via activating autophagy.

The close correlation between autophagy and insulin resistance has been confirmed. The deficient autophagy contributes to insulin resistance in obesity (Yang et al. 2010). In addition, the decreased levels of autophagyrelated proteins were shown in the liver tissues of mice with insulin resistance (Liu et al. 2009; Yang et al. 2010). Cai et al. showed that autophagy ablation led to restrained insulin action and general insulin resistance in mice (Cai et al. 2018). On the contrary, it was reported that autophagy hyperactivation significantly raised insulin sensitivity in mice treated with high fat diet (Yamamoto et al. 2018). In this study, EA treatment inhibited DHEA-induced insulin resistance as confirmed by decreasing serum insulin level and HOMA-IR index, and this effect was reversed by 3-MA. GLUT-4 is crucial for the transport of glucose to cells in response to insulin. It has been shown that the expression of GLUT-4 was reduced in skeletal muscles of diabetic patients with insulin resistance (Gaster et al. 2001). In addition, insulininduced AKT activation contributes to the cellular glucose uptake, which is attenuated when insulin resistance occurs (Cai et al. 2018; Yang et al. 2010). It has been documented that the phosphorylation of ERK may be stimulated by insulin independent of AKT pathway (Frendo-Cumbo et al. 2019). In our study, in response to insulin the GLUT4, p-AKT, and p-ERK levels in the skeletal muscle were attenuated in DHEA-challenged group. Although a slight change in these protein levels was observed affected by EA and 3-MA, there were no significant differences among groups. EA intervention restored GLUT4 translocation in response to insulin in PCOS-like rats, which was suppressed by 3-MA administration. More repeated experiments are needed to verify these results. Nevertheless, these findings indicated 
that EA improved insulin resistance in PCOS-like rats via activating autophagy.

The mTOR kinase has been recognized as a downstream regulator for insulin signaling (Laplante and Sabatini 2012). 4E-BP1, a target of mTOR kinase, can be phosphorylated by mTOR kinase activation. The frequent activation of mTOR has close relation with insulin resistance in patients and animals. Suppression of mTOR activity has been demonstrated to enhance insulin sensitivity (Um et al. 2004). Moreover, the activation of mTOR/4E-BP1 signaling may result in autophagy inhibition in multiple diseases (Follo et al. 2019; Wang and Zhang 2019). Therefore, mTOR/4E-BP1 signaling pathway may participate in the regulation of autophagymediated insulin resistance. In line with these observations, our results suggested that EA treatment effectively repressed DHEA-induced hyper-activation of mTOR/ 4E-BP1 signaling pathway in the skeletal muscle tissues of PCOS-like rats, indicating that EA alleviated insulin resistance in PCOS-like rats via inactivation of $\mathrm{mTOR} /$ 4E-BP1 signaling pathway.

Recent researches have indicated that mitochondrial dysfunction accelerates the progression of PCOS. For example, Reddy et al. discovered that mitochondria mass was evidently decreased in PCOS (Reddy et al. 2019). In addition, it was verified that mitochondrial dysfunction led to excess production of ROS and accelerated PCOS progression (Ding et al. 2017). More importantly, the mitochondrial copy number was remarkably decreased in PCOS patient with insulin resistance, indicating that mitochondrial dysfunction corelated with PCOS-IR (Ding et al. 2017; Saeed et al. 2019). In the current study, EA treatment effectively attenuated mitochondrial dysfunction in the ovarian tissue of PCOS-like rats. It was revealed that the restoration of mitophagy improved mitochondrial dysfunction and relieved heart failure (Shirakabe et al. 2016). Consistently, our data showed that EA-mediated improvement in mitochondrial dysfunction was partly reversed when autophagy was inhibited. Therefore, our findings suggested that EA attenuated mitochondrial dysfunction via promoting autophagy in the ovarian tissue of PCOS-like rats.

It has been recognized that ER stress is one of pathogenic mechanisms of PCOS (Azhary et al. 2019). ER stress is a biological process that maintains homeostasis, and many molecules and transcription factors are involved in ER stress. The levels of ER stress-related proteins GRP78, ATF4, and CHOP were up-regulated in PCOS patients (Banuls et al. 2017; Takahashi et al. 2017). GRP78 is a marker of ER stress, and the elevated GRP78 level suggests a severe protein misfolding condition. According to our results, an increased expression of GRP78, ATF4, and CHOP in the ovarian tissues of PCOS-like rats was verified. Just as we expected, EA treatment could down-regulate the increased GRP78, ATF4, and CHOP levels in the ovarian tissues of PCOSlike rats. Besides, a close relationship between ER stress and autophagy has been reported by previous studies (Cai et al. 2016; Cybulsky 2017). In this study, our findings demonstrated that inhibition of autophagy by 3-MA partly reversed EA-induced relief of ER stress. These findings revealed that autophagy-mediated ER stress attenuation was involved in the beneficial effect of EA on PCOS-like rats.

We aware of the fact that there are some limitations in the present study. The results indicated that EA and 3MA treatment had whole body effects, for example altering testosterone, FSH, LH, and insulin levels. Besides, the effects of EA on skeletal muscle and ovary tissues of rats have been studied, but the regulation of autophagy and ER stress in LH/FSH ratio in the brain and insulin resistance in other organs (liver and adipose tissue) has not been investigated in this study. Secondly, a preventing approach of EA was performed in this study. It is better to compare the effect and its related mechanisms of EA between treatment and pretreatment. In our future study, all these issues need to be further explored, which can more comprehensively elucidate the therapeutic mechanisms of EA in treating PCOS.

\section{Conclusions}

Our results demonstrated that EA treatment alleviated PCOS-like symptoms in rats via activating autophagy in skeletal muscle. In addition, activation of autophagy participated in EA-mediated improvement in insulin resistance, mitochondrial dysfunction and ER stress. Mechanistically, EA ameliorated autophagy deficiencymediated insulin resistance via inactivation of mTOR/ 4E-BP1 signaling pathway. Our results uncover the novel mechanisms of EA underlying the treatment of PCOS.

\section{Abbreviations \\ PCOS: Polycystic ovary syndrome; EA: Electroacupuncture; \\ DHEA: Dehydroepiandrosterone; ER: Endoplasmic reticulum; \\ mTOR: Mechanistic target of rapamycin kinase; SD: Sprague-Dawley; \\ FSH: Follicle stimulating hormone; LH: Luteal hormone; HOMA- \\ IR: Homeostasis model assessment of insulin resistance; PPARy: Peroxisome proliferator-activated receptor gamma; PGC-1a: PPARycoactivator-1a; 4E- \\ BP1: 4E-binding protein 1; GRP78: Glucose regulated protein 78; \\ ATF4: Activating transcription factor 4; CHOP: C/EBP-homologous protein}

\section{Acknowledgements}

Not applicable.

\section{Authors' contributions}

$Y P, L G, X Y$ were involved in the conception and design of the research, collected data, performed the statistical analysis, drafted and revised the manuscript. AG, BS, YR, JC contributed to acquisition, analysis, or interpretation of data for the work. All authors agreed to the publication. The authors read and approved the final manuscript.

\section{Funding}

This study was supported by grants from the National Natural Science Foundation of China (No. 81704114 and 81602272), the Postdoctoral Science 
Foundation of China (No. 2018 M640315), the Project of Excellent Innovation Talents by Heilongjiang University of Chinese Medicine (No. 2018RCD26), and the Postdoctoral Funding Project of Heilongjiang Provincial Government (No. LBH-Z18272).

\section{Availability of data and materials}

The datasets used or analyzed during the current study are available from the corresponding author on reasonable request.

\section{Ethics approval and consent to participate}

All animal experiments were performed in accordance with the Guidelines for the Care and Use of Laboratory Animals and approved by the ethics committee of Heilongjiang University of Chinese Medicine.

\section{Consent for publication}

Not applicable.

\section{Competing interests}

The authors declare that they have no competing interests.

\section{Author details}

'Disease Prevention Center, The First Affiliated Hospital, Heilongjiang University of Chinese Medicine, Harbin 150040, People's Republic of China. ${ }^{2}$ Department of Gynecological Oncology, Cancer Hospital of Harbin Medical University, Harbin 150081, People's Republic of China. ${ }^{3}$ Department of Radiation oncology, Cancer Hospital of Harbin Medical University, Harbin 150081, People's Republic of China. ${ }^{4}$ Graduate School, Heilongjiang University of Chinese Medicine, Harbin 150040, People's Republic of China. ${ }^{5}$ Department of Obstetrics and Gynecology, The First Affiliated Hospital, Heilongjiang University of Chinese Medicine, Harbin 150040, People's Republic of China.

\section{Received: 29 April 2020 Accepted: 13 July 2020}

Published online: 22 July 2020

\section{References}

Amir M, Czaja MJ. Autophagy in nonalcoholic steatohepatitis. Expert Rev Gastroenterol Hepatol. 2011;5(2):159-66. https://doi.org/10.1586/egh.11.4.

Anderson E, Lee MT, Lee GY. Cystogenesis of the ovarian antral follicle of the rat: ultrastructural changes and hormonal profile following the administration of dehydroepiandrosterone. Anat Rec. 1992;234(3):359-82. https://doi.org/10. 1002/ar.1092340307.

Azhary JMK, Harada M, Kunitomi C, Kusamoto A, Takahashi N, Nose E, et al. Androgens Increase Accumulation of Advanced Glycation End Products in Granulosa Cells by Activating ER Stress in PCOS. Endocrinology. 2020;161(2) doi:bqaa015 [pii]. https://doi.org/10.1210/endocr/bqaa0155724441.

Azhary JMK, Harada M, Takahashi N, Nose E, Kunitomi C, Koike H, et al. Endoplasmic reticulum stress activated by androgen enhances apoptosis of Granulosa cells via induction of death receptor 5 in PCOS. Endocrinology. 2019;160(1):119-32. https://doi.org/10.1210/en.2018-00675 5171869 [pii].

Banuls C, Rovira-Llopis S, Martinez de Maranon A, Veses S, Jover A, Gomez M, et al. Metabolic syndrome enhances endoplasmic reticulum, oxidative stress and leukocyte-endothelium interactions in PCOS. Metabolism. 2017;71:15362. doi: S0026-0495(17)30073-2 [pii]. https://doi.org/10.1016/j.metabol.2017. 02.012.

Batista JG, Soares JM Jr, Maganhin CC, Simoes RS, Tomaz G, Baracat EC. Assessing the benefits of rosiglitazone in women with polycystic ovary syndrome through its effects on insulin-like growth factor 1, insulin-like growth factorbinding protein-3 and insulin resistance: a pilot study. Clinics (Sao Paulo). 2012;67(3):283-7. doi: S1807-59322012000300014 [pii]. https://doi.org/10. 6061/clinics/2012(03)14.

Benrick A, Kokosar M, Hu M, Larsson M, Maliqueo M, Marcondes RR, et al. Autonomic nervous system activation mediates the increase in whole-body glucose uptake in response to electroacupuncture. FASEB J. 2017;31(8):328897. https://doi.org/10.1096/fj.201601381Rfj.201601381R.

Benrick A, Maliqueo M, Johansson J, Sun M, Wu X, Manneras-Holm L, et al. Enhanced insulin sensitivity and acute regulation of metabolic genes and signaling pathways after a single electrical or manual acupuncture session in female insulin-resistant rats. Acta Diabetol. 2014;51(6):963-72. https://doi.org/ 10.1007/s00592-014-0645-4.
Cai J, Pires KM, Ferhat M, Chaurasia B, Buffolo MA, Smalling R, et al. Autophagy ablation in adipocytes induces insulin resistance and reveals roles for lipid peroxide and Nrf2 signaling in adipose-liver crosstalk. Cell Rep. 2018;25(7): 1708-17 e1705. doi: S2211-1247(18)31629-2 [pii]. https://doi.org/10.1016/j. celrep.2018.10.040.

Cai Y, Arikkath J, Yang L, Guo ML, Periyasamy P, Buch S. Interplay of endoplasmic reticulum stress and autophagy in neurodegenerative disorders. Autophagy. 2016;12(2):225-44. https://doi.org/10.1080/15548627.2015.1121360.

Cao Y, Chen H, Zhao D, Zhang L, Yu X, Zhou X, et al. The efficacy of Tung's acupuncture for sex hormones in polycystic ovary syndrome: a randomized controlled trial. Complement Ther Med. 2019;44:182-8. doi: S09652299(19)30145-1 [pii]. https://doi.org/10.1016/j.ctim.2019.04.016.

Cybulsky AV. Endoplasmic reticulum stress, the unfolded protein response and autophagy in kidney diseases. Nat Rev Nephrol. 2017;13(11):681-96. https:// doi.org/10.1038/nrneph.2017.129 nrneph.2017.129 [pii].

Dai G, Lu G. Different protein expression patterns associated with polycystic ovary syndrome in human follicular fluid during controlled ovarian hyperstimulation. Reprod Fertil Dev. 2012;24(7):893-904. https://doi.org/10. 1071/RD11201RD11201 [pii].

Ding Y, Xia BH, Zhang CJ, Zhuo GC. Mutations in mitochondrial tRNA genes may be related to insulin resistance in women with polycystic ovary syndrome. Am J Transl Res. 2017;9(6):2984-96.

Follo C, Vidoni C, Morani F, Ferraresi A, Seca C, Isidoro C. Amino acid response by Halofuginone in Cancer cells triggers autophagy through proteasome degradation of mTOR. Cell Commun Signal. 2019;17(1):39. https://doi.org/10. 1186/s12964-019-0354-210.1186/s12964-019-0354-2 [pii].

Frendo-Cumbo S, Jaldin-Fincati JR, Coyaud E, Laurent EMN, Townsend L, Tan JMJ, et al. Deficiency of the autophagy gene ATG16L1 induces insulin resistance through KLHL9/KLHL13/CUL3-mediated IRS1 degradation. J Biol Chem. 2019. doi:jbc. RA119.009110 [pii]. https://doi.org/10.1074/jbc.RA119.009110.

Gaster M, Staehr P, Beck-Nielsen H, Schroder HD, Handberg A. GLUT4 is reduced in slow muscle fibers of type 2 diabetic patients: is insulin resistance in type 2 diabetes a slow, type 1 fiber disease? Diabetes. 2001;50(6):1324-9. https:// doi.org/10.2337/diabetes.50.6.1324.

Go KL, Lee S, Zendejas I, Behrns KE, Kim JS. Mitochondrial dysfunction and autophagy in hepatic ischemia/reperfusion injury. Biomed Res Int. 2015;2015: 183469. https://doi.org/10.1155/2015/183469.

Goodarzi MO, Dumesic DA, Chazenbalk G, Azziz R. Polycystic ovary syndrome: etiology, pathogenesis and diagnosis. Nat Rev Endocrinol. 2011;7(4):219-31. https://doi.org/10.1038/nrendo.2010.217 nrendo.2010.217 [pii].

Johansson J, Feng Y, Shao R, Lonn M, Billig H, Stener-Victorin E. Intense electroacupuncture normalizes insulin sensitivity, increases muscle GLUT4 content, and improves lipid profile in a rat model of polycystic ovary syndrome. Am J Physiol Endocrinol Metab. 2010;299(4):E551-9. https://doi. org/10.1152/ajpendo.00323.2010ajpendo.00323.2010 [pii].

Johansson J, Redman L, Veldhuis PP, Sazonova A, Labrie F, Holm G, et al. Acupuncture for ovulation induction in polycystic ovary syndrome: a randomized controlled trial. Am J Physiol Endocrinol Metab. 2013;304(9):E93443. https://doi.org/10.1152/ajpendo.00039.2013 ajpendo.00039.2013 [pii].

Kokosar M, Benrick A, Perfilyev A, Nilsson E, Kallman T, Ohlsson C, et al. A single bout of Electroacupuncture remodels epigenetic and transcriptional changes in adipose tissue in polycystic ovary syndrome. Sci Rep. 2018;8(1):1878. https://doi.org/10.1038/s41598-017-17919-5 [pii].

Laplante M, Sabatini DM. mTOR signaling in growth control and disease. Cell. 2012;149(2):274-93. https://doi.org/10.1016/j.cell.2012.03.017500928674(12)00351-0 [pii].

Lee GY, Croop JM, Anderson E. Multidrug resistance gene expression correlates with progesterone production in dehydroepiandrosterone-induced polycystic and equine chorionic gonadotropin-stimulated ovaries of prepubertal rats. Biol Reprod. 1998;58(2):330-7. https://doi.org/10.1095/ biolreprod58.2.330.

Lee WS, Yoo WH, Chae HJ. ER stress and autophagy. Curr Mol Med. 2015;15(8): 735-45. doi: CMM-EPUB-70543 [pii]. https://doi.org/10.2174/ 1566524015666150921105453

Legro RS, Barnhart HX, Schlaff WD, Carr BR, Diamond MP, Carson SA, et al. Clomiphene, metformin, or both for infertility in the polycystic ovary syndrome. N Engl J Med. 2007;356(6):551-66. doi:356/6/551 [pii]. https://doi. org/10.1056/NEJMoa063971.

Li D, You Y, Bi FF, Zhang TN, Jiao J, Wang TR, et al. Autophagy is activated in the ovarian tissue of polycystic ovary syndrome. Reproduction. 2018;155(1):85-92. https://doi.org/10.1530/REP-17-0499REP-17-0499 [pii]. 
Li M, Li L, Wang K, Su W, Jia J, Wang X. The effect of electroacupuncture on proteomic changes in the motor cortex of 6-OHDA Parkinsonian rats. Brain Res. 2017;1673:52-63. doi: S0006-8993(17)30326-8 [pii]. https://doi.org/10. 1016/j.brainres.2017.07.027.

Li X, Qi J, Zhu Q, He Y, Wang Y, Lu Y, et al. The role of androgen in autophagy of granulosa cells from PCOS. Gynecol Endocrinol. 2019;35(8):669-72. https:// doi.org/10.1080/09513590.2018.1540567.

Liu HY, Han J, Cao SY, Hong T, Zhuo D, Shi J, et al. Hepatic autophagy is suppressed in the presence of insulin resistance and hyperinsulinemia: inhibition of FoxO1-dependent expression of key autophagy genes by insulin. J Biol Chem. 2009;284(45):31484-92. https://doi.org/10.1074/jbc M109. 033936 M109.033936 [pii].

Liu J, Wu DC, Qu LH, Liao HQ, Li MX. The role of mTOR in ovarian neoplasms, polycystic ovary syndrome, and ovarian aging. Clin Anat. 2018:31(6):891-8. https://doi.org/10.1002/ca.23211.

Luchetti CG, Solano ME, Sander V, Arcos ML, Gonzalez C, Di Girolamo G, et al. Effects of dehydroepiandrosterone on ovarian cystogenesis and immune function. J Reprod Immunol. 2004;64(1-2):59-74. doi: S0165037804000695 [pii]. https://doi.org/10.1016/j.jri.2004.04.002.

Maliqueo M, Benrick A, Alvi A, Johansson J, Sun M, Labrie F, et al. Circulating gonadotropins and ovarian adiponectin system are modulated by acupuncture independently of sex steroid or beta-adrenergic action in a female hyperandrogenic rat model of polycystic ovary syndrome. Mol Cell Endocrinol. 2015;412:159-69. https://doi.org/10.1016/j.mce.2015.04.026 S03037207(15)00227-0 [pii]

Manneras L, Jonsdottir IH, Holmang A, Lonn M, Stener-Victorin E. Low-frequency electro-acupuncture and physical exercise improve metabolic disturbances and modulate gene expression in adipose tissue in rats with dihydrotestosterone-induced polycystic ovary syndrome. Endocrinology. 2008;149(7):3559-68. https://doi.org/10.1210/en.2008-0053 en.2008-0053 [pii].

Moran L, Teede H. Metabolic features of the reproductive phenotypes of polycystic ovary syndrome. Hum Reprod Update. 2009;15(4):477-88. https:// doi.org/10.1093/humupd/dmp008 dmp008 [pii].

Reddy TV, Govatati S, Deenadayal M, Sisinthy S, Bhanoori M. Impact of mitochondrial DNA copy number and displacement loop alterations on polycystic ovary syndrome risk in south Indian women. Mitochondrion. 2019:44:35-40. doi: S1567-7249(17)30088-0 [pii]. https://doi.org/10.1016/j.mito.2017.12.010.

Rosenfield RL, Ehrmann DA. The pathogenesis of polycystic ovary syndrome (PCOS): the hypothesis of PCOS as functional ovarian Hyperandrogenism revisited. Endocr Rev. 2016;37(5):467-520. https://doi.org/10.1210/er.2015-1104.

Saeed N, Hamzah $1 \mathrm{H}$, Al-Gharrawi SAR. Polycystic ovary syndrome dependency on mtDNA mutation; copy Number and its association with insulin resistance. BMC Res Notes. 2019;12(1):455. https://doi.org/10.1186/s13104-019-4453-3 [pii].

Shi Y, Li L, Zhou J, Sun J, Chen L, Zhao J, et al. Efficacy of electroacupuncture in regulating the imbalance of $\mathrm{AMH}$ and $\mathrm{FSH}$ to improve follicle development and hyperandrogenism in PCOS rats. Biomed Pharmacother. 2019;113: 108687. doi: S0753-3322(18)38304-5 [pii]. https://doi.org/10.1016/j.biopha. 2019.108687.

Shirakabe A, Zhai P, Ikeda Y, Saito T, Maejima Y, Hsu CP, et al. Drp1-dependent mitochondrial autophagy plays a protective role against pressure overloadinduced mitochondrial dysfunction and heart failure. Circulation. 2016; 133(13):1249-63. https://doi.org/10.1161/CIRCULATIONAHA.115.020502 CIRCULATIONAHA.115.020502 [pii].

Song X, Shen Q, Fan L, Yu Q, Jia X, Sun Y, et al. Dehydroepiandrosterone-induced activation of mTORC1 and inhibition of autophagy contribute to skeletal muscle insulin resistance in a mouse model of polycystic ovary syndrome. Oncotarget. 2018;9(15):11905-21. https://doi.org/10.18632/oncotarget.24190 24190 [pii].

Stener-Victorin E, Baghaei F, Holm G, Janson PO, Olivecrona G, Lonn M, et al. Effects of acupuncture and exercise on insulin sensitivity, adipose tissue characteristics, and markers of coagulation and fibrinolysis in women with polycystic ovary syndrome: secondary analyses of a randomized controlled trial. Fertil Steril. 2012;97(2):501-8. https://doi.org/10.1016/j.fertnstert.2011.11. 010 S0015-0282(11)02764-6 [pii].

Stener-Victorin E, Maliqueo M, Soligo M, Protto V, Manni L, Jerlhag E, et al. Changes in $\mathrm{HbA} 1 \mathrm{C}$ and circulating and adipose tissue androgen levels in overweight-obese women with polycystic ovary syndrome in response to electroacupuncture. Obes Sci Pract. 2016;2(4):426-35. https://doi.org/10.1002/ osp4.78 OSP478 [pii].

Stener-Victorin E, Zhang H, Li R, Friden C, Li D, Wang W, et al. Acupuncture or metformin to improve insulin resistance in women with polycystic ovary syndrome: study protocol of a combined multinational cross sectional casecontrol study and a randomised controlled trial. BMJ Open. 2019:9(1): e024733. https://doi.org/10.1136/bmjopen-2018-024733 bmjopen-2018024733 [pii].

Stepto NK, Cassar S, Joham AE, Hutchison SK, Harrison CL, Goldstein RF, et al. Women with polycystic ovary syndrome have intrinsic insulin resistance on euglycaemic-hyperinsulaemic clamp. Hum Reprod. 2013;28(3):777-84. https:// doi.org/10.1093/humrep/des463 des463 [pii].

Sumarac-Dumanovic M, Apostolovic M, Janjetovic K, Jeremic D, Popadic D, Ljubic $A$, et al. Downregulation of autophagy gene expression in endometria from women with polycystic ovary syndrome. Mol Cell Endocrinol. 2017;440:11624. doi: S0303-7207(16)30469-5 [pii]. https://doi.org/10.1016/j.mce.2016.11. 009.

Takahashi N, Harada M, Hirota Y, Nose E, Azhary JM, Koike H, et al. Activation of Endoplasmic Reticulum Stress in Granulosa Cells from Patients with Polycystic Ovary Syndrome Contributes to Ovarian Fibrosis. Sci Rep. 2017;7(1): 10824. https://doi.org/10.1038/s41598-017-11252-7 [pii].

Um SH, Frigerio F, Watanabe M, Picard F, Joaquin M, Sticker M, et al. Absence of S6K1 protects against age- and diet-induced obesity while enhancing insulin sensitivity. Nature. 2004;431(7005):200-5. https://doi.org/10.1038/nature02866 nature02866 [pii].

Wang H, Zhang G. Activation of CaMKKbeta-AMPK-mTOR pathway is required for autophagy induction by beta,beta-dimethylacrylshikonin against lung adenocarcinoma cells. Biochem Biophys Res Commun. 2019:517(3):477-83. doi: S0006-291X(19)31466-4 [pii]. https://doi.org/10.1016/j.bbrc.2019.07.100.

Wang Q, Deng H, Cheng K, Huang Z, Yin X, Zhou Y, et al. Manual acupuncture for the infertile female with polycystic ovary syndrome (PCOS): study protocol for a randomized sham-controlled trial. Trials. 2019;20(1):564. https:// doi.org/10.1186/s13063-019-3667-y [pii].

Wang $Y$, Wen L, Zhou S, Zhang $Y$, Wang XH, He YY, et al. Effects of four weeks intermittent hypoxia intervention on glucose homeostasis, insulin sensitivity, GLUT4 translocation, insulin receptor phosphorylation, and Akt activity in skeletal muscle of obese mice with type 2 diabetes. PLoS One. 2018;13(9): e0203551. https://doi.org/10.1371/journal.pone.0203551 PONE-D-18-16816 [pii].

Yamamoto S, Kuramoto K, Wang N, Situ X, Priyadarshini M, Zhang W, et al. Autophagy differentially regulates insulin production and insulin sensitivity. Cell Rep. 2018;23(11):3286-99. doi: S2211-1247(18)30778-2 [pii]. https://doi. org/10.1016/j.celrep.2018.05.032.

Yang L, Li P, Fu S, Calay ES, Hotamisligil GS. Defective hepatic autophagy in obesity promotes ER stress and causes insulin resistance. Cell Metab. 2010; 11(6):467-78. https://doi.org/10.1016/j.cmet.2010.04.005 S15504131(10)00116-6 [pii]

Zain MM, Jamaluddin R, Ibrahim A, Norman RJ. Comparison of clomiphene citrate, metformin, or the combination of both for first-line ovulation induction, achievement of pregnancy, and live birth in Asian women with polycystic ovary syndrome: a randomized controlled trial. Fertil Steril. 2009; 91(2):514-21. https://doi.org/10.1016/j.fertnstert.2007.12.002 S00150282(07)04184-2 [pii].

Zeng Q, He H, Wang XB, Zhou YQ, Lin HX, Tan ZP, et al. Electroacupuncture preconditioning improves myocardial infarction injury via enhancing AMPKdependent autophagy in rats. Biomed Res Int. 2018;2018:1238175. https://doi. org/10.1155/2018/1238175

Zeng X, Huang Q, Long SL, Zhong Q, Mo Z. Mitochondrial Dysfunction in Polycystic Ovary Syndrome. DNA Cell Biol. 2020. https://doi.org/10.1089/dna. 2019.5172.

\section{Publisher's Note}

Springer Nature remains neutral with regard to jurisdictional claims in published maps and institutional affiliations. 